\title{
Study of Nanoscale Recorded Marks on Phase-Change Recording Layers and the Interactions With Surroundings
}

\author{
Shih Kai Lin ${ }^{1,2}$, I. Chun Lin ${ }^{1,2}$, Sen Yong Chen ${ }^{1,2}$, Hao Wen Hsu ${ }^{1,2}$, and Din Ping Tsai ${ }^{1,2}$ \\ ${ }^{1}$ Department of Physics, National Taiwan University, Taipei 10617, Taiwan, R.O.C. \\ ${ }^{2}$ Center for Nanostorage Research, National Taiwan University, Taipei 10617, Taiwan, R.O.C.
}

\begin{abstract}
Conductive-atomic force microscopy (C-AFM) has been used for studying nanoscale recorded marks with different length on the phase-change recording layer of optical disks. Through C-AFM images, a comparison of nanoscale recorded marks on phase-change recording layer under different writing strategies and writing power has been taken. The comparison can help analyze the combination of writing strategy, writing power and laser pulse width. The various lengths of recorded marks for high density data storage have also been found out. The interactions between phase-change recording layers and their surroundings have also been studied. This study opens up a possibility to improve the capacity of data storage in today's commercial optical disks.
\end{abstract}

Index Terms-Conductive-atomic force microscopy (C-AFM), DVD, optical disk, phase-change recording layer, recorded mark, writing strategy.

\section{INTRODUCTION}

$\mathbf{F}$ OR YEARS, a variety of studies pertaining to high density data storage techniques such as Blu-ray disks, high definition digital versatile disks (HD-DVD), and super-resolution near-field structure disks, have been propounded and improved. With the advance of these technologies, the size of recorded marks is becoming smaller and the way to exam the nanoscale recorded marks is becoming more difficult. Although scanning electron microscope (SEM) or transmission electron microscope (TEM) has been used for the examination of recorded marks, their processes are laborious. Thus, there is a need for an examination method with high resolution and rapidity to help the development of high density data storage technologies. Recently a method of fast imaging recorded marks on phase-change optical disks has been successfully developed and applied to studies of recording marks on the phase-change recording layer of optical disks [1], [2]. The method has provided us an opportunity for studying the recorded marks on phase-change rewriteable optical disks in depth, especially for marks on super-resolution near-field structure disks. Moreover, using the method, the methods to record marks within $100 \mathrm{~nm}$ has been found out. The approach is based on conductive-atomic force microscopy (C-AFM) and can be easily used for studying characterizations of the length, shape and the relation of writing strategies to formation of recorded marks. With all the knowledge and information of recorded marks on phase-change rewriteable optical disks, the study of characterizations of recorded marks on phase-change

Digital Object Identifier 10.1109/TMAG.2006.888471 rewriteable optical disks through different writing conditions can be achieved thoroughly.

\section{EXPERIMENTAL SETUP}

A dynamic optical disc tester (DDU-1000, Pulstec Inc., Japan) with laser wavelength of $658 \mathrm{~nm}$, and numerical aperture (NA) of 0.6) is employed to write recorded marks on commercial rewriteable DVD + RW disks. A static tester, a pump-probe laser system with two semiconductor laser diodes operating at $\lambda_{1}=633 \mathrm{~nm}$ (read), and $\lambda_{2}=658 \mathrm{~nm}$ (write), is used for forming marks on a phase-change recording layer coated on a glass by a RF-sputter [3]. C-AFM images of recorded marks written with different writing strategies and diverse writing laser power are scanned through two commercial scanning probe microscopes (SPM), Dimension-3100 SPM from Veeco Instrument (Santa Barbara, CA) and MFP-3D ${ }^{\mathrm{TM}}$ of Asylum Research. The scanning direction of C-AFM images is adjusted to be perpendicular to the tracking direction, and a DC bias voltage is applied to the recording layer of the disk via the silver paste, with a range from $1 \mathrm{mV}$ to $100 \mathrm{mV}$ (the probe is set to be virtual ground). The conductive-AFM probe coated with PtIr $_{5}$ on both sides of the cantilever (Model Pointprobe Cont-Pt) used in the experiments is manufactured by Nanosensors (Germany), and the resistivity of the probe is around $0.01 \Omega / \mathrm{cm}$ to $0.02 \Omega / \mathrm{cm}$. The C-AFM images are scanned at constant velocity of $10 \mu \mathrm{m} / \mathrm{s}$ in contact mode on the sample surface, and proper contact force is set for the topography image simultaneously.

\section{RESUlTS AND DISCUSSION}

Fig. 1 shows C-AFM images of recorded marks written with different laser power, $9 \mathrm{~mW}, 10 \mathrm{~mW}, 11 \mathrm{~mW}$, respectively. It also reveals that the writing power has serious influence on the size of recorded marks when the writing strategies are fixed 

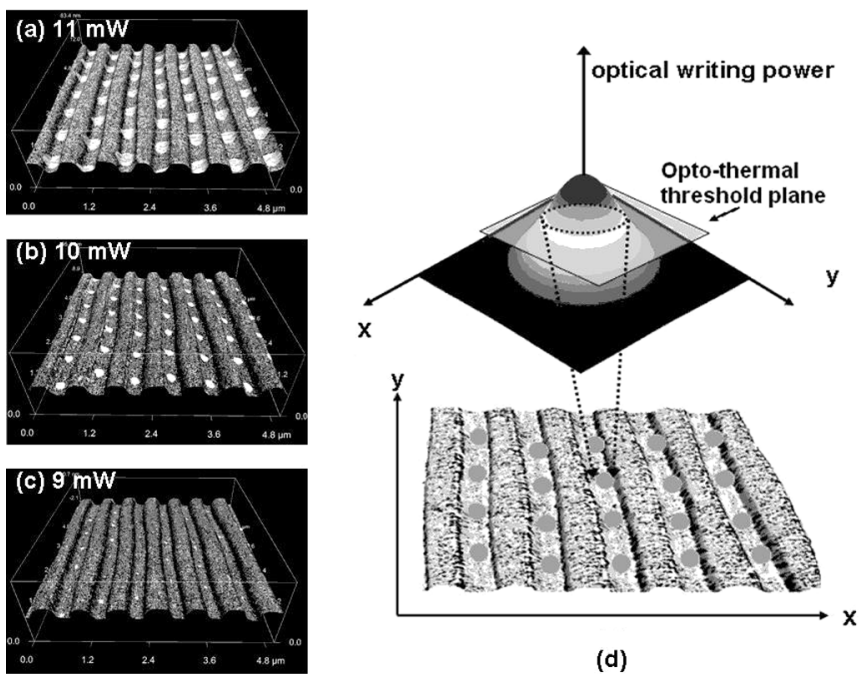

(d)

Fig. 1. Three-dimensional C-AFM images of recorded marks with respect to writing power and the illustration of opt-thermal effect on the size of recorded marks.

$(2 \mathrm{t} 14 \mathrm{t})$. In the experiment, $2 \mathrm{t}$ stands for $28 \mathrm{~ns}$, which corresponds to $100 \mathrm{~nm}$ under a linear velocity of $3.5 \mathrm{~m} / \mathrm{sec}$. There is a threshold power under which no recorded marks will be written. Thus, through adjusting writing laser power, above the threshold power, marks of various sizes can be written on phasechange rewriteable optical disks. The marks with a length below $100 \mathrm{~nm}$ in the experiment can be achieved.

Fig. 2 shows C-AFM images of recorded marks on phasechange recording layers written at different writing strategies with the same writing power, $9.5 \mathrm{~mW}$. In this figure, the erasing effect of writing laser to the last recorded marks can be observed. As the spacing between recorded marks reduces, the erasing effect between recorded marks is becoming obvious, especially at the writing strategy of $2 \mathrm{t} 2 \mathrm{t}$, shown in Fig. 2(a). The space between recorded marks can be adjusted through different writing strategies, as shown in Fig. 2(e). The erasing effect result in crescent recorded marks because the subsequent writing laser pulse erases part of the last recorded marks. The measured length of recorded marks on Fig. 2(a) is $134 \mathrm{~nm}$ on average, performing the potential for writing nanoscale recorded marks. The erasing effect is inconspicuous when the spacing between marks is large enough, and the shape of recorded marks remain elliptic, such as the situation of writing strategy of $2 \mathrm{t} 14 \mathrm{t}$, as shown in Fig. 2(c). Moreover, when the writing power goes up under the writing strategy of $2 \mathrm{t} 6 \mathrm{t}$, as shown in Fig. 2(d), the separate marks of Fig. 2(b) become connected. This result can be extended to explain the long marks formed on common commercial phase-change rewriteable optical disks. Several long marks can be seen if writing laser turns off for a longer period between any two writing duty cycles, as a cooling pulse width that has been reported.[4] If marks are written with higher power or a longer pulse, the shapes of marks will merely become a large ellipse, not long marks. Therefore, it suggests that for the implementation of next-generation optical storage, different lengths of marks, among which the shortest lengths is of subwavelength, can be achieved by adjusting both writing strategy and writing laser power. With the information of C-AFM images, one can
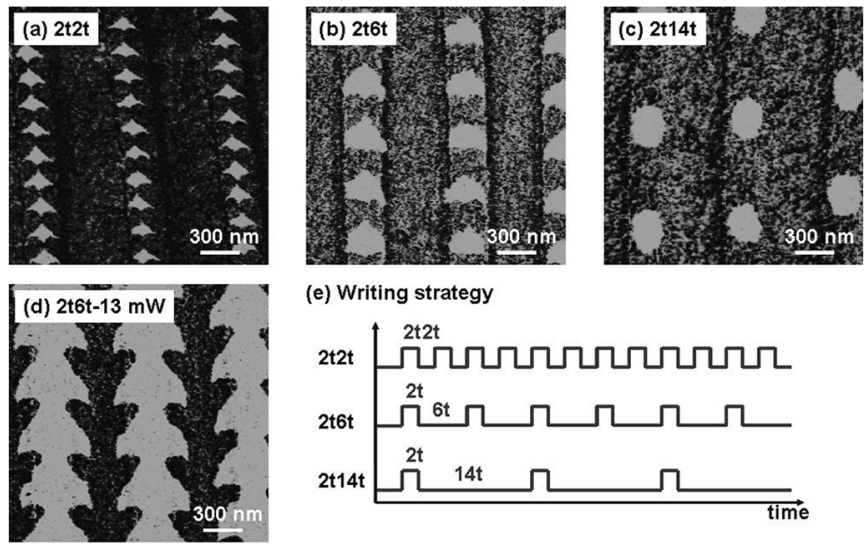

(e) Writing strategy

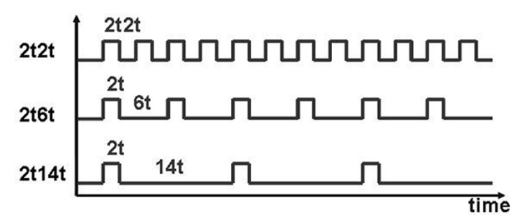

Fig. 2. (a)-(d) C-AFM images of recorded marks written with various writing strategies and writing power of $9.5 \mathrm{~mW}$ as well as $13 \mathrm{~mW}$. (e) An illustration of writing strategies.

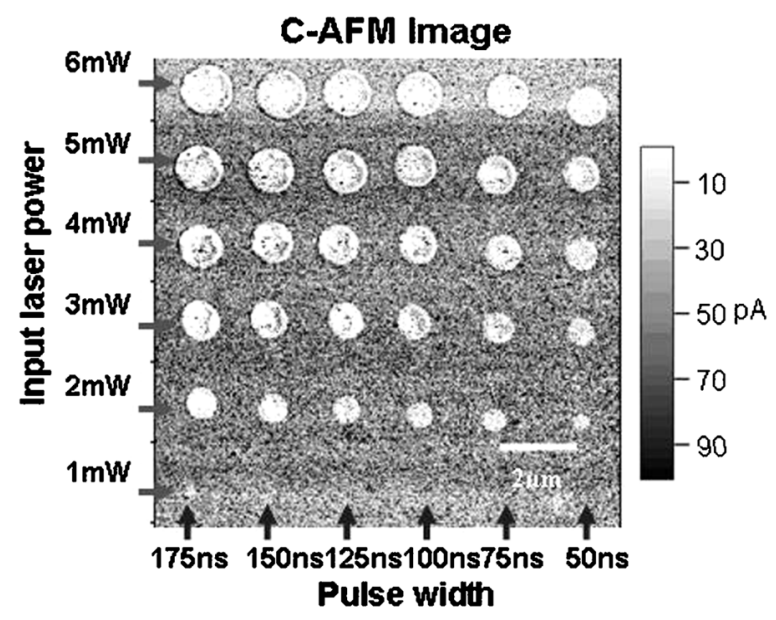

Fig. 3. C-AFM images of recorded marks on phase-change recording layer without protective layer written by a static tester(a pump-probe laser system) with different laser power and different duty cycle.

know that with appropriate writing strategies and writing power, marks of different lengths can be achieved.

In the study of recorded marks written on the phase-change recording layer, it is found that recording layers with different surroundings will affect the forming of recorded marks. Fig. 3 shows C-AFM image of an array of recorded marks written by using a static tester, a pump-probe laser system, on a phasechange recording layer coated on a glass without any protective layer. The marks are written with various input laser powers, 1-6 mW, and different pulse widths, 50-175 ns. The sizes of marks obviously decrease as the input laser power or pulse width decreases. However, the white areas in C-AFM image showing lower conductivity are not resulted from amorphous state of phase-change material, but stemmed from an area covered by an oxidation layer formed as the writing laser heats the phasechange recording layer. To accurately study the sizes and shapes in terms of writing powers or pulse widths, the oxidation should be eliminated. Notwithstanding the oxidation, the result still implies that small recorded marks can be acquired through altering writing laser power or/and input pulse width. To avoid the oxidation of the naked surface, a protective layer can be used to cover 
$P_{w w}=20 \mathrm{~mW}$

Q Topography
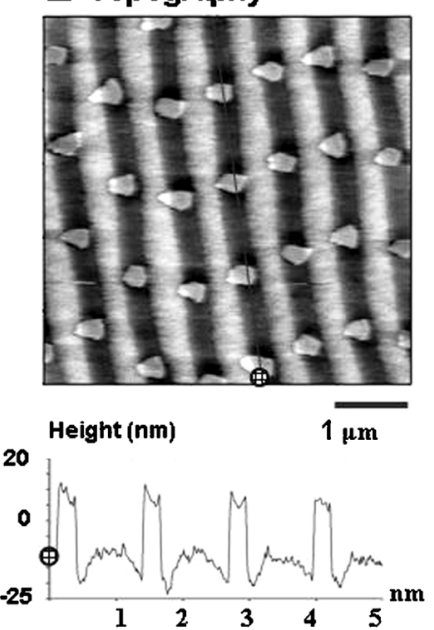
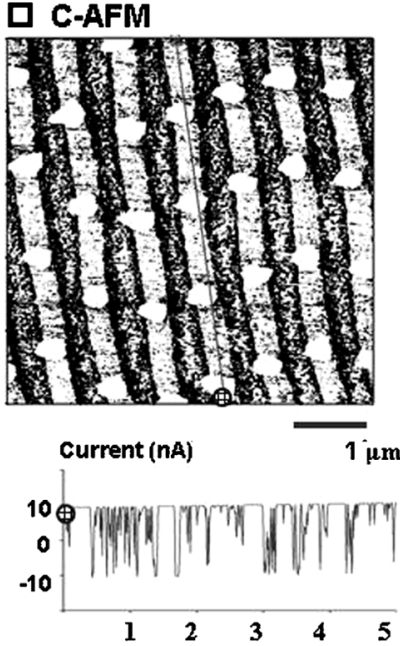

Fig. 4. AFM and C-AFM images of recorded marks on phase-change recording layer written under high laser power $(20 \mathrm{~mW})$.

the phase-change recording layer, such as optical disk. The results in Figs. 1 and 2 have been performed with an optical disk structure so as to prevent the recording layer from oxidation. Another advantage for an optical disk is that recorded marks can be written by using a dynamic optical disc tester.

However, it should be noted that when the written power is too high, there is damage between the recording layer and its adjacent protective layer, as shown in Fig. 4. From the AFM image in the left-hand side of Fig. 4, the damaged areas can be seen obviously and its height is measured to be about $20 \mathrm{~nm}$, shown in the cross section in the left-hand side of Fig. 4. From the C-AFM image in the right-hand side of Fig. 4, these damaged areas in white have lower conductivity, but actually they are not the amorphous marks, which also have lower conductivity.[5] The damage is resulted from the very high writing power, making part of the protective layer mix with part of the recording layer in the vicinity of the interface between these two layers. Then, as the protective layer was peeled to expose the recording layer, some residues of the protective layer adhere above the recorded marks. As a result, these areas covered with residues of protective layers result in lower conductivity. Even though the white areas in C-AFM image show good spatial order, they are not the

actual shape of recorded marks. Therefore, in the experiment, suitable laser power should be employed to avoid the adherence of other materials.

\section{CONCLUSION}

For the implementation of the next-generation data storage optical disks, it is essential to effectively reduce the size of recorded marks to increase the capacity of data storage. The C-AFM images of nanoscale recorded marks on phase-change rewriteable optical disks can provide detailed information of recorded marks, such as shapes and sizes. Through the study of recorded marks written with different writing strategies and writing power, the way to make appropriate lengths and shapes of recorded marks on phase-change rewriteable optical disks can be implemented, helping to determine what kind of writing strategy and how much the writing power should be taken for different kinds of optical disks.

\section{ACKNOWLEDGMENT}

This work was supported in part by the Ministry of Economic Affairs, R.O.C., under Project Number 95-EC-17-A-08-S1-0006, and the Center for Nano Science and Technology, National Taiwan University.

\section{REFERENCES}

[1] P. L. Yang, P. H. Chang, C. C. Hsu, W. C. Lin, T. S. Kao, C. W. Lin, and D. P. Tsai, "Advanced imaging of nanometer-scale recorded bits on super-resolution near-field optical disk," J. Korean Phys. Society, vol. 47, pp. S115-S118, Aug. 2005.

[2] S. K. Lin, P. Yang, I. C. Lin, H. W. Hsu, and D. P. Tsai, "Resolving nano scale recording bits on phase-change rewritable optical disks," Jpn. J. Appl. Phys., vol. 45, pp. 1431-1434, 2006.

[3] M. Mansuripur, J. K. Erwin, W. Bletscher, P. Khulbe, K. Sadeghi, X. Xun, A. Gupta, and B. Mendes, "Static tester for characterization of phase-change, dye polymer, and magneto-optical media for optical data storage," Appl. Opt., vol. 38, pp. 7095-7104, 1999.

[4] M. Miyamoto, A. Hirotsune, Y. Miyauchi, K. Ando, M. Terao, N. Tokusyuku, and R. Tamura, "Analysis of Mark-formation process for phase-change media," IEEE J. Select. Topics Quantum Electron., vol. 4, pp. 826-831, 1998.

[5] S. K. Lin, I. C. Lin, and D. P. Tsai, "Characterization of nano recorded marks at different writing strategies on phase-change recording layer of optical disks," Opt. Express, vol. 14, pp. 4452-4458, 2006.

Manuscript received August 10, 2006 (e-mail: dptsai@phys.ntu.edu.tw). 